\title{
A General Boundary Condition with Linear Flux for Advection-Diffusion Models ${ }^{\dagger}$
}

\author{
T.Y. MIYAOKA ${ }^{1 *}$, J.F.C.A. MEYER ${ }^{1}$ and J.M.R. SOUZA ${ }^{2}$
}

Received on November 21, 2016 / Accepted on April 19, 2017

\begin{abstract}
Advection-diffusion equations are widely used in modeling a diverse range of problems. These mathematical models consist in a partial differential equation or system with initial and boundary conditions, which depend on the phenomena being studied. In the modeling, boundary conditions may be neglected and unnecessarily simplified, or even misunderstood, causing a model not to reflect the reality adequately, making qualitative and/or quantitative analyses more difficult. In this work we derive a general linear flux dependent boundary condition for advection-diffusion problems and show that it generates all possible boundary conditions, according to the outward flux on the boundary. This is done through an integral formulation, analyzing the total mass of the system. We illustrate the exposed cases with applications willing to clarify their meanings. Numerical simulations, by means of the Finite Difference Method, are used in order to exemplify the different boundary conditions' impact, making it possible to quantify the flux along the boundary. With qualitative and quantitative analysis, this work can be useful to researchers and students working on mathematical models with advection-diffusion equations.
\end{abstract}

Keywords: boundary conditions, partial differential equations, mathematical models, computer simulation.

\section{INTRODUCTION}

The first application of the diffusion equation was done by Fourier in 1822 [1], when he proposed its use to model heat distribution. At that time, the main concern was to analyze the model for simple cases, including simple boundary conditions such as fixed concentrations at the boundaries. Once theory was developed, the diffusion equation can now be combined with advection (or convection) processes, resulting in advection-diffusion equations, which demand more elaborate boundary conditions, depending on the phenomena being modeled.

\footnotetext{
$\dagger^{\top}$ This work was supported by CAPES.

*Corresponding author: Tiago Yuzo Miyaoka - E-mail: tiagoyuzo@gmail.com

${ }^{1}$ Departamento de Matemática Aplicada, IMECC, Universidade Estadual de Campinas, R. Sérgio Buarque de Holanda, 651, 13083-859 Campinas, SP, Brasil. E-mail: joni@ime.unicamp.br

2 Departamento de Matemática, IMECC, Universidade Estadual de Campinas, R. Sérgio Buarque de Holanda, 651, 13083-859 Campinas, SP, Brasil. E-mail: jumarta@gmail.com
} 
In [2], the authors separate the applications of advection-diffusion models in two categories: inorganic, such as temperature and concentration of matter, and organic, populations of organisms, which is the main concern of their book. Amongst other interesting and rich examples, the authors cite the applications to diffusion of spores, insect pheromones, insect dispersal. An application to temperature and heat transfer can be found in [3]. Advection-diffusion models have been widely used in mathematical ecology. Applications include organic subjects, as in [2], and inorganic, in a way inspired by that of [4], modeling pollution dispersal. The main purpose of these works is to obtain models based on advection-diffusion equations or systems and numerical approximations to their solutions, generally by means of the Finite Elements or Finite Differences methods. Some works analyzed pollutant dispersal, either in rivers [5], in the sea [6], lakes in two [7] and three dimensions [8], air [9], or mixed [10]. In [11], steady-state air pollution is modeled. Other works treated the diffusion of interacting animals, as the change of habitat in fish [12], biological control of the boll weevil [13], biological control of the horn fly affecting beef cattle [14], and skipjack tuna movement in the western Pacific Ocean [15]. Other works studied the diffusion of population dynamics in the presence of pollutants, as in a two preys system [16], two predators and two preys system [17], two competitors [18], development of macroalgae [19], and the sediment impact in four benthic populations [20]. A different approach is the consideration of diffusion and migration (advection) in epidemiological compartmental models, as a capybara disease [21], foot-and-mouth disease [22], and avian influenza [23]. General population movement is also studied, aiming to compare different models and/or techniques, as in $[24,25,26,27]$. As each of these works has their own particularities, appropriate analyses at the boundaries are necessary in each case, but in most of the cited cases, the authors decided to make simple assumptions in order to obtain more tractable boundary conditions, sometimes due to lack of information about the studied phenomenon. One exception is the already mentioned work [6], in which the authors consider a boundary condition similar (but less general) to the one treated in this present work. The inappropriate use of boundary conditions can lead to models which do not reflect reality so well, or to different interpretations, disturbing qualitative and/or quantitative analyses. For the interpretations, we will be based on the previously cited works, considering mostly the pollution case, but sometimes analogies using the animals and heat cases will help us understand the model as a whole.

A simple analysis about boundary conditions in ecological problems modeled by advectiondiffusion equations can be found in [28], where the authors relate the outward flux with the concentration present on the boundary, but only for the homogeneous case. In the previously mentioned work [2], the authors consider boundary conditions for each specific model, lacking a general analysis. A derivation for flux conditions can be found in [29], but only for a specific application.

The present work aims to derive and analyze a general boundary condition with linear flux for the advection-diffusion equation. By general we mean that all boundary conditions with linear flux are particular cases from this general one. Our analysis aims to clarify its meanings and usefulness in each of its particular cases, as much as in the general case. 
In Section 2, we derive a general boundary condition with linear flux for an advection-diffusion equation, through an integral formulation. Integrating the concentration under study over the spatial domain, we obtain the total mass of the system, to which the analyses are made, in each of its particular cases. In Section 3, numerical simulations with the Finite Difference Method are made and mass by time graphs are shown for several particular boundary conditions. Additionally, a convergence analysis for numerical errors is made. The discretization and a brief algorithm is shown in Appendix 4. Conclusions are presented in Section 4.

Considering an initial-boundary value problem in $u=u(\vec{x}, t), \vec{x}=\left(x_{1}, \ldots, x_{n}\right) \in \mathbb{R}^{n}$, we have in literature [29] three different boundary condition types: Dirichlet, Neumann and Robin, each of these being separated in homogeneous, if it does not involve values beyond $u$, or non homogeneous, if it does. These conditions can be found in Table 1, where $u$ is the solution of the boundary value problem, $f, g$, and $h$ are arbitrary functions and $a$ and $b$ arbitrary parameters, which may or may not depend on $(\vec{x}, t)$. A Dirichlet, or first kind, condition specifies the value of $u$ on the boundary, either being zero or any function $f$ that may depend or not on other variables. A Neumann, or second kind, condition, on the other hand, specifies the derivative of the solution $u$ along the boundary, more precisely the directional derivatives in the direction of the external unitary normal vector $\vec{n}$, as also shown in Table 1 . A Robin, or third kind, condition involves both the value of $u$ and its derivative, specifying an equation that must be valid along the boundary, as in the last two rows of Table 1 . In the next sections, we will consider only two spatial variables $(x, y)$, but all the analyses are analogous to one or three, or even $n$ dimensional problems.

Table 1: Boundary Condition Types.

\begin{tabular}{lll}
\hline Condition & & Kind \\
\hline$u=0$ & homogeneous & Dirichlet or first kind \\
$u=f(\vec{x}, t)$ & non homogeneous & \\
\hline$\frac{\partial u}{\partial \vec{n}}=0$ & non homogeneous & \\
$\frac{\partial u}{\partial \vec{n}}=g(\vec{x}, t)$ & homogeneous & \\
\hline$a u+b \frac{\partial u}{\partial \vec{n}}=0$ & & Robin or third kind \\
$a u+b \frac{\partial u}{\partial \vec{n}}=h(\vec{x}, t)$ & non homogeneous & \\
\hline
\end{tabular}

\section{MATHEMATICAL MODELING OF BOUNDARY CONDITIONS}

Lets consider a substance being diffused, under effect of a velocity field, by influence of a current for example, in a river, lake, or a portion of the sea, with or without reaction, decay, external 
sources, among others, as in some of the previously cited works. Also consider a population of animals spreading in a territory, such as its natural habitat or a confined space, with some preference in its movement, which generates a transport effect, plus other possible influences such as vital dynamics. Both, substance and animals, can be modeled by the advection-diffusion equation $[2,28]$ :

$$
\frac{\partial u}{\partial t}+\nabla \cdot(-\alpha(x, y) \nabla u+\mathbb{V}(x, y) u)=f(u, x, y, t)
$$

Where $u$ denotes the concentration of substance or animals, with mass/distance ${ }^{2}$ units; the $\nabla$ operator is calculated in spatial variables $x$ and $y ; \alpha(x, y)$ is the diffusion coefficient, with distance $^{2} /$ time units; $\mathbb{V}(x, y)=(v(x, y), w(x, y))$ is the velocity or migration field with distance/time units; and $f(u, x, y, t)$ is an external concentration source with mass/(distance ${ }^{2}$ time) units, which includes reaction, dynamic terms, depending or not on $u$. For simplicity we will consider $f$ identically null, as it does not alter the analyses on the boundary. A similar approach with $f$ different from zero can be found in [29]. The spatial domain, the water medium or animal habitat, is an open bounded region $\Omega \in \mathbb{R}^{2}$ with boundary $\partial \Omega$ and the temporal interval is given by $I=\left(t_{0}, t_{f}\right]$. Besides that, $u\left(x, y, t_{0}\right)=u_{0}(x, y)$ is an arbitrary initial condition.

This problem can be simplified to a stationary one in a direct way, considering the temporal derivative as zero in (2.1). All boundary analyses are applicable to the stationary case in the same way as in the temporal one.

Another situation, where a temperature diffuses in a thin metal plate, with an external source of heat represented by $f$, can also be modeled by equation (2.1), but with the advection term null, $\mathbb{V}(x, y)=0$.

\subsection{General Boundary Condition Derivation}

In order to obtain the most general boundary condition to this equation, we integrate (2.1) over the whole domain $\Omega$, at an arbitrary time instant $t \in I$, already considering $f(u, x, y, t)$ identically null:

$$
\iint_{\Omega} \frac{\partial u}{\partial t} d A+\iint_{\Omega} \nabla \cdot(-\alpha \nabla u+\mathbb{V} u) d A=0
$$

Considering $u(x, y, t)$ uniformly continuous in $t$, we can take the temporal derivative out of the integral. Applying the Divergence Theorem to the second term, one obtains:

$$
\frac{\partial}{\partial t} \iint_{\Omega} u(x, y, t) d A+\oint_{\partial \Omega}(-\alpha \nabla u+\mathbb{V} u) \cdot \vec{n} d s=0 .
$$

We observe that $\iint_{\Omega} u(x, y, t) d A=M(t)$, where $M(t)$ is the total mass of $u$ inside the domain $\Omega$ for each $t \in I$. The equation (2.2) may then be written depending on the total mass variation rate:

$$
\frac{d M(t)}{d t}+\oint_{\partial \Omega}(-\alpha \nabla u+\mathbb{V} u) \cdot \vec{n} d s=0
$$


This way, if we want the total mass variation of the system to be null, i.e., that there is no incoming nor outgoing concentration in the domain, then the temporal derivative in (2.3) should be null and:

$$
\oint_{\partial \Omega}(-\alpha \nabla u+\mathbb{V} u) \cdot \vec{n} d s=0
$$

The only way for this integral to be zero for any solution $u$, is if the integrand itself is zero. This integrand is the outward normal component of the flux of the concentration $u$ over the domain, $J(u)=-\alpha \nabla u+\mathbb{V} u$. The term $-\alpha \nabla u$ denotes the flux due to diffusion and $\mathbb{V} u$ the flux due to advection. Rearranging the terms, observing that $\nabla u \cdot \vec{n}$ is the directional derivative of $u$ in the normal direction, the outward flux must be null, for $(x, y) \in \partial \Omega$ and $t \in I$ :

$$
-\alpha \frac{\partial u}{\partial \vec{n}}+\mathbb{V} \cdot \vec{n} u=0 .
$$

This is the null flux boundary condition for (2.1). We wish to obtain a condition in which the exit/entrance flux is not zero, but dependent on factors external to the domain and/or concentration density on the boundary. For this purpose, we equal the flux at left hand side of (2.4) to a linear combination of $u$ and an external source, obtaining the following general linear flux boundary condition, for $(x, y) \in \partial \Omega$ and $t \in I$ :

$$
-\alpha \frac{\partial u}{\partial \vec{n}}+\mathbb{V} \cdot \vec{n} u=\beta(u-c)+\gamma c .
$$

Where $c=c(x, y, t)$ represents an external source of concentration, such as $f(u, x, y, t)$ but acting only on the boundary and independent of $u$. $\beta$ and $\gamma$ are parameters, which might or not depend on $(x, y)$ and $t$, and relate the entrance/exit rate of concentration on the boundary with $u$ and $c$ densities. Both $\beta$ and $\gamma$ have distance/time units and $c$ has mass/distance ${ }^{2}$ units. In this work we consider $\beta$ and $\gamma$ as constants, and positive unless specified, for didactic purposes, but the extension is straightforward. The word "linear" means that the flux along the boundary depends linearly on $u$. Writing this condition in another way:

$$
-\alpha \frac{\partial u}{\partial \vec{n}}+\mathbb{V} \cdot \vec{n} u=\beta u+(\gamma-\beta) c .
$$

We can then see that the flux dependent on $u$ is proportional to $\beta$, while the external flux, due to $c$, is proportional to $\gamma-\beta$. We may have $\beta, \gamma$, and/or $c=0$, each situation providing particular cases. Varying these combinations we have all possible boundary conditions with linear flux. The particular linear combination at the right hand side of (2.4) or (2.6) was carefully taken in order to model the fluxes of both $u$ and external source $c$ along the boundary, in a general point of view. Applications and examples are exhibited in the next sections.

To analyze the meaning of each term of the right hand side of condition (2.4) or (2.6), we return to the total mass system analysis, but this time imposing the generalized condition (2.5) directly upon equation (2.3), obtaining:

$$
\frac{d M(t)}{d t}=-\oint_{\partial \Omega} \beta u d s-\oint_{\partial \Omega} \gamma c d s+\oint_{\partial \Omega} \beta c d s .
$$

Tend. Mat. Apl. Comput., 18, N. 2 (2017) 
The line integrals in this equation represent $u$ and $c$ masses on the boundary of the domain. These masses are proportional to rates $\beta$ and $\gamma$, which are influencing the total mass variation. Considering $\beta>0, \gamma>0$ and $c>0$, we have escape of concentration $u$ with rate $\beta$, escape of concentration $c$ with rate $\gamma$, and entrance of concentration $c$ with rate $\beta$. This can be seen schematically in Figure 1. If the signs of $\beta, \gamma$ or $c$ were exchanged, their entrances and escapes would need to be inverted.

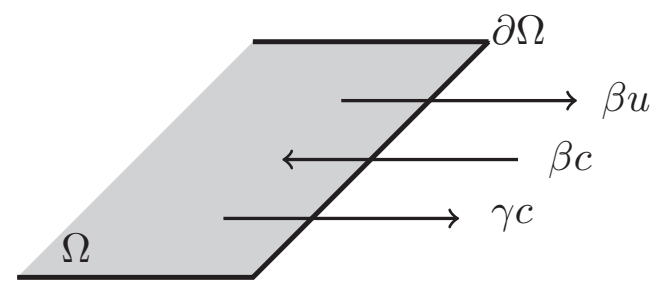

Figure 1: Flux illustration for equation (2.1) with boundary condition (2.5). If $\beta>0, \gamma>0$, and $c>0$, the flux $\beta u$ and $\gamma c$ are outward, while the flux $\beta c$ is inward.

For a better analysis of this condition, in the following we will divide it in distinct cases for $\beta$, considering $\beta \rightarrow \infty, \beta=0$, and $\beta \neq 0, \beta<\infty$, in each of these cases considering $\gamma, c$ and $\mathbb{V}$ null or not. A summary can be found in Table 2 . As previously said, these options covers all possible boundary conditions with linear flux for equation (2.1).

\subsection{Flux independent of $u, \beta=0$}

\subsubsection{External source, $\gamma \neq 0$ and $c \neq 0$}

In this case we consider $\beta=0$, i.e., we do not consider flux dependent upon $u$. So (2.5) turns into:

$$
-\alpha \frac{\partial u}{\partial \vec{n}}+\mathbb{V} \cdot \vec{n} u=\gamma c
$$

This non homogeneous Robin condition relates the flux with the external source $c$ by means of the rate $\gamma$. In this case we have a constant, or at least not dependent on $u$, concentration removal in the system (or injection, if $\gamma c<0$ ).

An example could be with $u$ as a pollution, and $c$ acting by removing pollution on the boundary from inside to outside the domain, regardless of the quantity $u$ that is already on the domain. Or, if $\gamma c<0$ so the flux is positive in the outward direction, it would be an external source of concentration from outside the boundary to inside the domain.

\subsubsection{Null flux, $\gamma=0$ and/or $c=0$}

If either $\gamma$ or $c$ are also null, we should have no external source:

$$
-\alpha \frac{\partial u}{\partial \vec{n}}+\mathbb{V} \cdot \vec{n} u=0 .
$$


Table 2: Summary of boundary conditions for equation (2.1)

Condition
$-\alpha \frac{\partial u}{\partial \vec{n}}+\mathbb{V} \cdot \vec{n} u=\gamma c$
$-\alpha \frac{\partial u}{\partial \vec{n}}+\mathbb{V} \cdot \vec{n} u=0$
$\frac{\partial u}{\partial \vec{n}}=\gamma c$
$\frac{\partial u}{\partial \vec{n}}=0$
$-\alpha \frac{\partial u}{\partial \vec{n}}+\mathbb{V} \cdot \vec{n} u=\beta(u-c)$
$-\alpha \frac{\partial u}{\partial \vec{n}}+\mathbb{V} \cdot \vec{n} u=\beta u+(\gamma-\beta) c$
$-\alpha \frac{\partial u}{\partial \vec{n}}+\mathbb{V} \cdot \vec{n} u=\beta u$
$-\alpha \frac{\partial u}{\partial \vec{n}}=\beta(u-c)$
$-\alpha \frac{\partial u}{\partial \vec{n}}=\beta(u-c)+\gamma c$
$-\alpha \frac{\partial u}{\partial \vec{n}}=\beta u$
$u=0$

Which is the same homogeneous Robin condition as (2.4), with no flux and the total mass of the system constant, as discussed before. We can interpret this equation as an equality between the normal components of diffusion flux and advection flux, in order to maintain the concentration exchange null.

Considering $u$ as a pollutant concentration, this condition would mean that the boundary completely blocks the passage of the substance to outside the domain, and also blocks any outside substance from entering. If $u$ were a population of animals, this condition translates to a fence or natural limitation of the territory that keeps all the animals that are inside confined, and not letting any outsider enter, acting as a perfect barrier. Also, this condition could model a situation 
in which the value of $u$ is constant along the boundary, that is: everything that enters is equivalent to whatever leaves the domain, so that the flux is null.

\subsubsection{External source with no advection, $\gamma \neq 0, c \neq 0$ and $\mathbb{V}=0$}

In the particular case of the purely diffusive problem, $\mathbb{V}=0$, and condition (2.7) simplifies to a non homogeneous Neumann one:

$$
-\alpha \frac{\partial u}{\partial \vec{n}}=\gamma c
$$

In the purely diffusive problem the flux is given solely by the directional derivative (Fick's law [2]), so the interpretation is the same as of (2.7).

The pollutant case is also valid as an example here, not considering the transport by making $\mathbb{V}=0$. But another example can be the previously mentioned heat problem, with $u$ measuring the temperature in a metal plate and at the boundaries a fixed temperature being kept by some external source. Considering $u$ as the ambient temperature, the domain could be a room isolated from outer temperature, with a heater inside, represented by $c$.

\subsubsection{Null flux with no advection, $\gamma=0$ or $c=0$ and $\mathbb{V}=0$}

If $\gamma=0$ or $c=0$ in the purely diffusive case, we have the following homogeneous Neumann condition, which is a particular case of (2.8):

$$
\frac{\partial u}{\partial \vec{n}}=0 .
$$

Continuing with the temperature example, this condition means that the boundaries are perfectly isolated so that there is no influence of the external media to the inside temperature. This may occur in a metal plate or in the ambient temperature examples, without external influence.

We observe that this condition is a no flux one only for the purely diffusive case, and if applied to the general problem (2.1), it will neglect the advective flux $\mathbb{V} \cdot \vec{n} u$. This would mean that $\beta u=\mathbb{V} \cdot \vec{n} u$, with $\beta \neq 0$, considering a flux due to $\beta u$ that actually does not exist. This condition may be interesting if the boundary in study is a barrier to diffusion, so the diffusive flux will be null, but not a barrier to advection, leaving its flux unaltered.

\subsection{Partial flux, $\beta \neq 0$}

\subsubsection{Exchange between inside and outside, $\gamma=0$ and $c \neq 0$}

Before proceeding to the general case, in which all the parameters in (2.5) are different from zero, we will consider the case in which only $\gamma=0$, i.e., only the rate due to external source is taken as zero. Then we have the following non homogeneous Robin condition:

$$
-\alpha \frac{\partial u}{\partial \vec{n}}+\mathbb{V} \cdot \vec{n} u=\beta(u-c) .
$$


This condition tells us that parameter $\beta$ relates the flux exchange, exit or entrance, of both $u$ and $c$ on the boundary. That is, if $u>c$, with $\beta>0$, then concentration $u$ along the boundary is greater than the external source $c$, therefore the flux is positive to the outside and this difference is balanced. If $u<c$, with $\beta>0$, then the external source is greater than the concentration on the boundary, and then the flux is negative as well as the sign of $\beta(u-c)$, leading to an inward flux.

In the pollutant example, this condition models an exchange of concentration on the boundary, depending on both external concentration, $c$, and internal, $u$, as explained in the previous paragraph, as an osmosis phenomenon, greater concentrations tend to migrate to locations with lower concentrations. Considering $u$ as confined animals, this condition would mean that they can leave and enter along the boundary, not freely, but accordingly to the concentration present there. So the animals would leave the domain if the concentration were grater than $c$, or enter if it were smaller than $c$.

\subsubsection{Dependence on $u$ and external source, $\gamma \neq 0$ and $c \neq 0$}

This is the most important condition, because it includes all others. It is a non homogeneous Robin condition and arises when all the parameters in (2.5) are non zero. We rewrite it here in two different ways for convenience:

$$
\begin{aligned}
& -\alpha \frac{\partial u}{\partial \vec{n}}+\mathbb{V} \cdot \vec{n} u=\beta u+(\gamma-\beta) c . \\
& -\alpha \frac{\partial u}{\partial \vec{n}}+\mathbb{V} \cdot \vec{n} u=\beta(u-c)+\gamma c .
\end{aligned}
$$

Looking at (2.12a) we can divide the flux in two parts: one depending on $u$ and the other on $c$. The greater the concentration of $u$ on the boundary, the greater will be the exit (or entrance) of it, being $\beta$ the rate that regulates this change. If $\beta>0$, we have entrance, and if $\beta<0$, we have exit of $u$ from the domain. Also, the flux relative to $(\gamma-\beta) c$ does not vary with $u$, but it can depend on $x, y, t$ if $\gamma, \beta$ and/or $c$ do so. The sign of $\gamma-\beta$ tells us if there is input into $(\gamma>\beta)$, or output from the domain $(\gamma<\beta)$ of external source $c$.

On the other hand, looking at $(2.12 \mathrm{~b})$, we can see that the term $\gamma c$ is a constant injection of concentration as in (2.7), which is related to factors external to the domain. Also, the term $\beta(u-$ $c$ ) is the same as in (2.11), having here the same role. So we see that $\beta$ is a parameter that relates the inside of the domain to the outside, while $\gamma$ does not depend on the inside, considering only the contribution of external factors.

Considering again $u$ as a concentration pollutant, this condition considers the exchange dependent on the external concentration, as in (2.11), but also with a constant removal as in (2.7) (or constant source if $\gamma c<0$ ). 


\subsubsection{Dependence on $u, \gamma=0$ and $c=0$}

In the case $\gamma=\beta$, or $c=0,(2.11)$ becomes a homogeneous Robin condition:

$$
-\alpha \frac{\partial u}{\partial \vec{n}}+\mathbb{V} \cdot \vec{n} u=\beta u .
$$

This condition relates the flux only with the density along the boundary, without external factors. If $\beta>0$, this condition means that the flux is outward positive so, as $u$ increases, the exit of concentration from the domain becomes higher. If $\beta<0$, we have the opposite. We note that this is a particular case of (2.11) without an external balance factor, so that the behavior of the flux does not depend on the outside of the domain.

An example could be either the pollutant or the animals case, in a boundary which allows passage, similar to that of (2.11) but without contributions from the outside. According to [28], this is the standard boundary condition for equation (2.1).

\subsubsection{Dependence on $u, \gamma=\beta, c \neq 0$}

In this case, the condition also reduces to (2.13), but there is a conceptual difference: the external source $c$ is not null, but the equality between the rates $\gamma$ and $\beta$ causes the whole term $(\gamma-\beta) c$ in (2.12a) to vanish, so that there is an equality between the entrance and exit of $c$ in the domain: $\beta c=\gamma c$ (which is entrance or exit depends on the signs involved).

This situation is highly unlikely because the model is an approximation of the reality, therefore parameters considered do not have precise values. A possible example could be, in the pollutant case, an artificial regulation of $\gamma$, in order to precisely cause the equality $\gamma=\beta$, if one can control the entrance/exit of the pollutant.

\subsubsection{Exchange between inside and outside with no advection, $\gamma=0, c \neq 0$ and $\mathbb{V}=0$}

Considering now the purely diffusive case, $\mathbb{V}=0$, and $\gamma=0$, we have:

$$
-\alpha \frac{\partial u}{\partial \vec{n}}=\beta(u-c),
$$

with same interpretation as in (2.11). The example in (2.11) about the pollutant is also valid here, but a typical example is the temperature case, this condition being known as the heat radiation boundary [3], where there is heat exchange with the external media. In this fashion, $c$ represents the external temperature, and the boundary condition balances the difference in the temperature from inside the domain and outside, with the same analysis as in (2.11).

\subsubsection{Dependence on $u$ and external source with no advection, $\gamma \neq 0, c \neq 0$ and $\mathbb{V}=0$}

In the purely diffusive case, $\mathbb{V}=0$, the general linear condition is:

$$
-\alpha \frac{\partial u}{\partial \vec{n}}=\beta(u-c)+\gamma c
$$


which is a particular case of (2.12), just without the advection term, so the same interpretation is valid. Again considering $u$ as the ambient temperature in a room, this combines the radiation boundary in (2.14) and the external source in (2.9), so that there is an exchange of temperature with the exterior of the room and an internal source of heat.

\subsubsection{Dependence on $u$ with no advection, $\gamma=0$ or $c=0$ and $\mathbb{V}=0$}

If, besides $\mathbb{V}=0$, either $\gamma=0$ or $c=0$, we have an homogeneous Robin condition:

$$
-\alpha \frac{\partial u}{\partial \vec{n}}=\beta u,
$$

which is a particular case of (2.13).

In the heat diffusing in a metal plate, this means that there is change of temperature in the extremes of the plate, which does not depend on the outside, but only on the temperature along the boundary.

\subsection{Total flux, $\beta \rightarrow \infty$}

\subsubsection{Total flux with external source, $c \neq 0$}

Rewriting condition (2.5) in order to isolate $u-c$, we obtain:

$$
\frac{1}{\beta}\left(-\alpha \frac{\partial u}{\partial \vec{n}}+\mathbb{V} \cdot \vec{n} u-\gamma c\right)=u-c .
$$

Increasing the flow due to the presence of $u$ in the boundary indefinitely, i.e. considering unlimited growth of $\beta$, so that $\beta \rightarrow \infty$, we have the following non homogeneous Dirichlet condition:

$$
u=c .
$$

This tells us that all concentration $u$ that arrives on the boundary dissipates, because the flux due to $\beta$ is unlimited, thereby the value of $u$ is completely described by the external source $c$.

In the pollution example, the unlimited growth of $\beta$ means that all the pollution arriving on the boundary crosses it completely, or is totally absorbed, so that the only concentration of pollutant that remains there is due to the external source $c$.

\subsubsection{Total flux without external source, $c=0$}

If besides $\beta \rightarrow \infty$ we also have $c=0$, we obtain the homogeneous Dirichlet condition, which also has total exit flux but without external source, keeping the concentration zero along the boundary:

$$
u=0 \text {. }
$$


In the pollution case, we also have a complete passage of concentration, but without any source, so that the concentration remains zero on the boundary. In a population dispersal case, this condition can be used in a situation in which no animal can stay along the boundary, because of the terrain, for example.

We note that the same conditions, (2.17) or (2.18) are valid if $\alpha=0, \mathbb{V}=0$, or even $\gamma=0$, thereby the total flux condition is independent of these quantities.

\subsection{Mixed boundary conditions}

In practical situations, mathematical models often consider domains with heterogeneous boundaries. This means that the boundary $\partial \Omega$ must be separated in several regions $\partial \Omega_{i}, i=1, \ldots, N$, with $\partial \Omega=\cup_{i=1}^{N} \partial \Omega_{i}$, the union being disjoint. Each of these regions $\partial \Omega_{i}$ can then be modeled by any of the presented boundary conditions (2.7)-(2.18). Most of the already mentioned works utilize this kind of mixed boundary conditions in their models. In particular, in [10] the authors explain each part of the boundary considered for an air/lake pollution model.

\section{NUMERICAL SIMULATIONS}

In order to obtain numerical approximations to solutions of problem (2.1), we utilized second order Finite Difference formulas in spatial variables and Crank-Nicolson Method in the time variable [30]. For simplicity we considered a rectangular domain $\Omega=[0, L] \times[0, H]$. For boundary points we applied the finite difference formulas directly in the boundary conditions obtaining complementary equations. We then obtained an algebraic linear system to be solved at each time step, which is solved by the $L U$ factorization method. As the system matrix is constant, it may be factorized only once. Matlab was used for the implementation of the computational routines. Details about the numerical solutions calculations can be found in the Appendix.

The illustrative parameters utilized were $\alpha=0.1 ; \mathbb{V}=(0.02,0.02) ; \beta=0, \beta=0.1, \beta=0.05$, or $\beta=100 ; \gamma=0, \gamma=0.1$ or $\gamma=0.05 ; c=0, c=1$ or $c=-1$. The length and height of the domain were $L=H=1$, and the time interval $J=(0,2]$. The domain was discretized using $2^{6}$ intervals in both $x$ and $y$ direction and $2^{10}$ in time. The initial condition was $u_{0}(x, y)=\kappa \exp \left(-64\left((x-0.5)^{2}+(y-0.5)^{2}\right)\right)$, a gaussian at the center of the domain, where $\kappa$ is a constant to maintain initial mass equal to the unit. This gaussian was chosen because it has negligible value on the boundary of the domain. Therefore at the beginning of the simulations, the boundary is unaffected. As one can obtain any of the exposed boundary conditions from (2.5), this general expression was used in the implementation, and the parameters adjusted to obtain the different conditions.

Our computational experiments aim to illustrate some of the cases exposed in the previous section, focusing on the total mass of the system as a function of time, $M(t)$. This mass is calculated by numerical integration, with repeated Simpson's formula, using the numerical solution obtained. More precisely, we obtain numerical approximations to the solutions of problem (2.1), with boundary conditions (2.7), (2.8), (2.11), (2.12), (2.13), (2.17), and (2.18), Figure 2. 


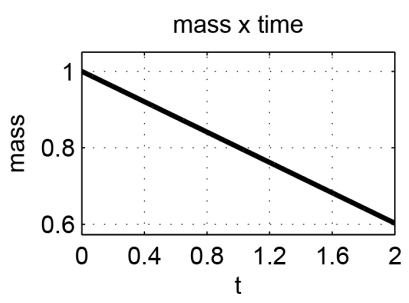

(a) $\beta=0, \gamma=0.05, c=1$

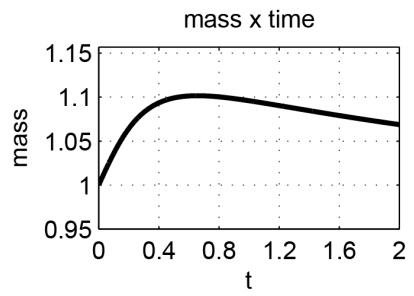

(d) $\beta=0.1, \gamma=0, c=1$

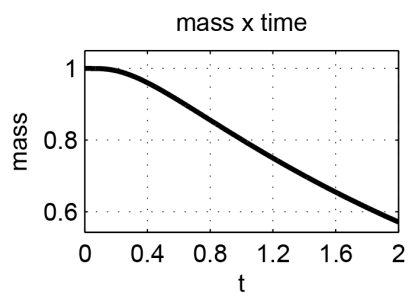

(g) $\beta=0.1, \gamma=0, c=0$

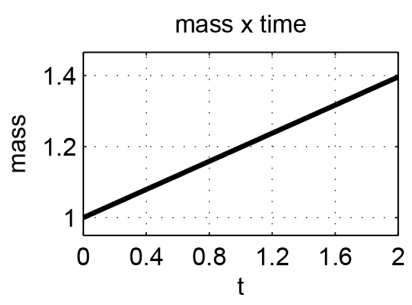

(b) $\beta=0, \gamma=0.05, c=-1$

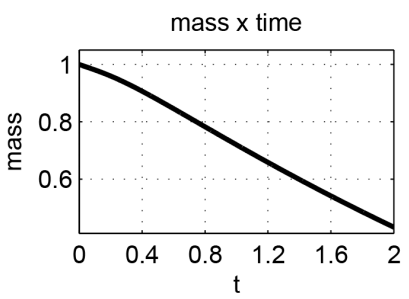

(e) $\beta=0.05, \gamma=0.1, c=1$

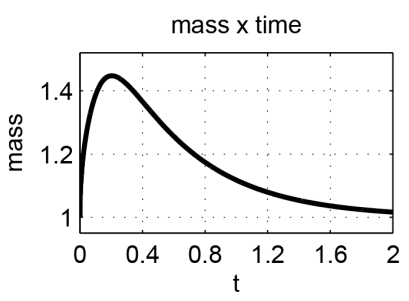

(h) $\beta=100, \gamma=0, c=1$

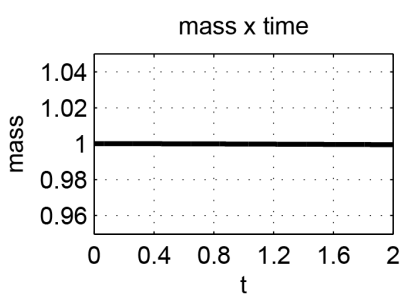

(c) $\beta=0, \gamma=0, c=0$

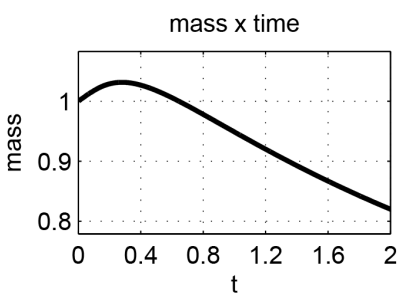

(f) $\beta=0.1, \gamma=0.05, c=1$

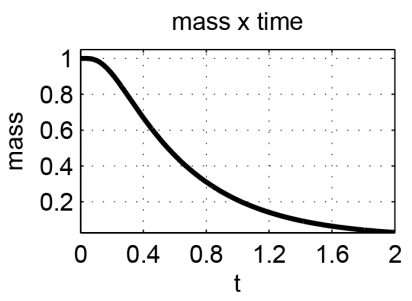

(i) $\beta=100, \gamma=0, c=0$

Figure 2: Mass by time graphs in numerical solutions of problem (2.1), for boundary conditions (2.7): 2(a), 2(b), (2.8): 2(c), (2.11): 2(d), (2.12): 2(e), 2(f), (2.13): 2(g), (2.17): 2(h), and (2.18): 2(i).

The non-simulated conditions are particular cases from these but for the purely diffusive case, so that their analyses are the same.

In Figure 2(a) the parameters were $\beta=0, \gamma=0.05$, and $c=1$. We have condition (2.7) with $\gamma c>0$, which implies in outward linear flux along the boundary. The flux is constant, given by $\gamma c$, therefore the mass decreases linearly.

In Figure 2(b) the parameters were $\beta=0, \gamma=0.05, c=-1$. We have condition (2.7) with $\gamma c<0$, which implies in inward linear flux on the boundary. The flux is constant, therefore the mass increases linearly.

In Figure 2(c) the parameters were $\beta=0, \gamma=0, c=0$. We have condition (2.8), with flux equal to zero. There is no flux neither outward nor inward the boundary, therefore the mass is constant through all time. 
In Figure 2(d) the parameters were $\beta=0.1, \gamma=0, c=1$. We have condition (2.11), with exchange between internal and external concentration, given by $u$ and $c$ respectively. At the beginning there is inward flux, because $u<c$ on the boundary, therefore the mass increases. But after (approximately at $t=0.6$ ), the flux becomes outward, as the concentration along the boundary increases making $u>c$, and the mass decreases.

In Figure 2(e) the parameters were $\beta=0.05, \gamma=0.1, c=1$. We have condition (2.12), with the combined effects of (a) and (d). Therefore the flux is always outward because of the term $\gamma c$, but it starts small and increases because of $\beta(u-c)$. The mass always decreases.

In Figure 2(f) the parameters were $\beta=0.1, \gamma=0.05, c=1$. We have condition (2.12), but with $\beta<\gamma$, therefore the flux due to $c$ in inward, causing the concentration to increase at the beginning. As the system mass increases, so does the term $\beta u$, and the flux becomes outward (approximately at $t=0.3$ ), causing the mass to decrease.

In Figure 2(g) the parameters were $\beta=0.1, \gamma=0, c=0$. We have condition (2.13), with flux dependent only on $u$. As in the very beginning there is no concentration on the boundary, the mass remains constant. But as concentration increases along the boundary, so does the outward flux, causing the mass to decrease.

In Figure 2(h) the parameters were $\beta=100, \gamma=0, c=1$. We have condition (2.17), with total flux is outward the domain, and also a total injection due to $c$. At the beginning there is an increase in the mass, because there is only injection. Afterwards there is a decrease in concentration due to the total outward flux.

In Figure 2(i) the parameters were $\beta=100, \gamma=0, c=0$. We have condition (2.18), with total flux outward and no external source. The flux becomes higher as the concentration starts to cross the boundary causing the mass to decrease.

In Tables 3 and 4 we show convergence tests for our numerical solutions. In each table we show absolute and relative errors in max norm obtained comparing solutions in consecutive meshes, which consist in regular subdivisions of the domain. In Table $3, n$ denotes the number of divisions in $x$ and $y$ directions, which are equal, and in Table $4, n$ is the number of divisions in time $t$. For the temporal convergence, we used the mass of the system $M(t)$ for the errors, which contains information of the whole spatial domain. We also show the numerical rate of convergence of the methods comparing two consecutive errors. As expected, both in space and time the rates obtained converge to 2 , since the methods used have $\Delta x^{2}, \Delta y^{2}$ and $\Delta t^{2}$ orders. In Figure 3 we show an error by mesh grid sizes loglog graph for both spatial and temporal analyses, with linear fits for each case. These results collaborate to the accuracy of the results obtained.

\section{CONCLUSION}

In this work, we show that one unique general boundary condition with linear flux, equation (2.5), generates all other particular cases. We also show, by means of qualitative analysis and numerical simulations, the interpretations and importance of boundary conditions in different situations in 
Table 3: Spatial errors and rates of convergence for (2.1) numerical solutions.

\begin{tabular}{rccc}
\hline \multicolumn{1}{c}{$n$} & Error & Relative error & Order \\
\hline 8 & & & \\
16 & $8.86 \times 10^{-1}$ & $5.35 \times 10^{-2}$ & \\
32 & $2.45 \times 10^{-1}$ & $1.50 \times 10^{-2}$ & 1.8565 \\
64 & $6.16 \times 10^{-2}$ & $3.79 \times 10^{-3}$ & 1.9889 \\
128 & $1.54 \times 10^{-2}$ & $9.50 \times 10^{-4}$ & 1.9988 \\
256 & $3.86 \times 10^{-3}$ & $2.38 \times 10^{-4}$ & 1.9998 \\
\hline
\end{tabular}

Table 4: Temporal errors and rates of convergence for (2.1) numerical solutions.

\begin{tabular}{rccc}
\hline \multicolumn{1}{c}{$n$} & Error & Relative error & Order \\
\hline 16 & & & \\
32 & $2.40 \times 10^{-7}$ & $2.40 \times 10^{-7}$ & \\
64 & $5.95 \times 10^{-8}$ & $5.95 \times 10^{-8}$ & 2.0098 \\
128 & $1.49 \times 10^{-8}$ & $1.49 \times 10^{-8}$ & 2.0024 \\
256 & $3.72 \times 10^{-9}$ & $3.72 \times 10^{-9}$ & 2.0006 \\
512 & $9.29 \times 10^{-10}$ & $9.29 \times 10^{-10}$ & 2.0002 \\
1024 & $2.32 \times 10^{-10}$ & $2.32 \times 10^{-10}$ & 2.0001 \\
2048 & $5.80 \times 10^{-11}$ & $5.80 \times 10^{-11}$ & 2.0021 \\
4096 & $1.45 \times 10^{-11}$ & $1.45 \times 10^{-11}$ & 2.0022 \\
\hline
\end{tabular}

advection-diffusion problems. The qualitative analyses made in section 2 show the variety of possible scenarios at the boundaries. Also, the quantitative analysis, made possible because of numerical simulations, illustrated by the system total masses in Figure 2, highlights the difference between each of the different cases and their consequences in the solution of the problems. The connection between the different cases was made by the general condition, equation (2.5), which facilitates the comprehension of boundary conditions as a whole and in each case separately.

In some works the boundary conditions in advection-diffusion models are simplified. Either because of lack of information about the true values for the parameters involved or in order to facilitate the numerical methods involved. For example, in some cases where the outward flux must be null, condition (2.10) is used instead of $(2.8)[5,7,8,9,10,12,13,14,16,17,18,19$, $20,21,23]$. In others, where there is a partial flux on the boundary, condition (2.16) is used instead of $(2.13)$, [8, 10, 14, 20]. In general, these considerations are a good approximation for the numerical simulations because of the difficulties in finding estimations for the proper values of model parameters, but the qualitative meanings of the conditions are surely different. 


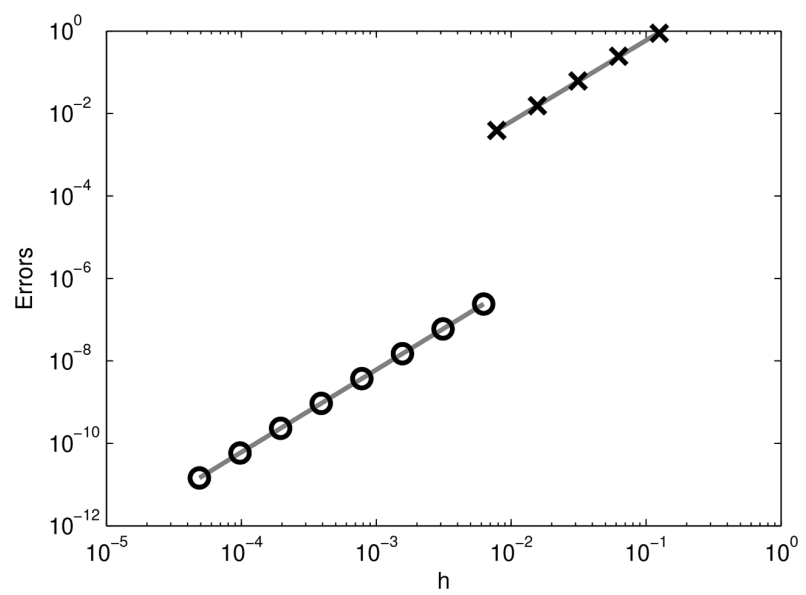

Figure 3: Numerical errors by spatial and temporal mesh grid sizes in loglog scale. Crosses denote spatial errors and circles temporal errors. In each case linear fits are presented.

The analyses made can, and should, be extended to non-linear cases, as the boundary condition modeled in [24]. Nevertheless, the system mass analysis could be made for any particular boundary condition, linear or not. Researchers and students working on mathematical models with advection-diffusion equations may use this work to help their understandings in modeling processes at the boundaries.

\section{ACKNOWLEDGEMENTS}

The authors thank the reviewers for corrections and suggestions.

RESUMO. Equações de Difusão-Advecção são amplamente utilizadas em modelagens de uma diversidade de problemas. Estes modelos matemáticos consistem em uma equação ou sistema de equações diferenciais parciais com condições iniciais e de contorno, que dependem dos fenômenos a serem estudados. Na modelagem, condições de contorno podem ser negligenciadas e desnecessariamente simplificadas, ou ainda mal entendidas, levando a um modelo não refletir bem a realidade, tornando análises qualitativas e/ou quantitativas mais difíceis. Neste trabalho nós deduzimos uma condição de contorno geral com fluxo linear para problemas de difusão-advecção e mostramos que ela gera todas as outras condições de contorno possíveis, de acordo com o fluxo externo à fronteira. Isso é feito via uma formulação integral, analisando a massa total do sistema. Nós ilustramos os casos expostos com aplicações na expectativa de elucidar seus significados. Simulações numéricas, por meio do Método de Diferenças Finitas, são utilizadas para exemplificar o impacto de diferentes condições de contorno, tornando possível quantificar o fluxo na fronteira. Com as análises qualitativas e 
quantitativas, este trabalho pode ser útil a pesquisadores e estudantes trabalhando em modelos matemáticos com equações de difusão-advecção.

Palavras-chave: condições de contorno, equações diferenciais parciais, modelos matemáticos, simulações computacionais.

\section{REFERENCES}

[1] J. Fourier. Theorie analytique de la chaleur, par M. Fourier. Paris: Chez Firmin Didot, père et fils, (1822).

[2] A. Okubo \& S.A. Levin. Diffusion and ecological problems: modern perspectives, vol. 14. New York: Springer Science \& Business Media, (2013).

[3] T.L. Bergman, F.P. Incropera, D.P. DeWitt \& A.S. Lavine. Fundamentals of heat and mass transfer. Jefferson City: John Wiley \& Sons, (2011).

[4] G.I. Marchuk. Mathematical models in environmental problems, vol. 16. North-Holland: Elsevier, (2011).

[5] D.C. Mistro. "O problema da poluição em rios por mercúrio metálico: modelagem e simulação numérica”. Master's thesis, DMA, IMECC, UNICAMP, Campinas, SP, (1992).

[6] J.F.C.A. Meyer, R.F. Cantão \& I.R.F. Poffo. "Oil spill movement in coastal seas: modelling and numerical simulations". WIT Trans. Ecol. Envir., 27 (1998), 23-32.

[7] E.C.C. Poletti \& J.F.C.A. Meyer. "Numerical methods and fuzzy parameters: an environmental impact assessment in aquatic systems". Comput. Appl. Math., pp. 1-12, (2016).

[8] A. Krindges. Modelagem e simulação computacional de um problema tridimensional de difusãoadvecção com uso de Navier-Stokes. PhD thesis, DMA, IMECC, UNICAMP, Campinas, SP, (2011).

[9] S.E.P. Castro. "Modelagem matemática e aproximação numérica do estudo de poluentes no ar". Master's thesis, DMA, IMECC, UNICAMP, Campinas, SP, (1993).

[10] J.F.C.A. Meyer \& G.L. Diniz. "Pollutant dispersion in wetland systems: Mathematical modelling and numerical simulation.” Ecol. Model., 200(3) (2007), 360-370.

[11] D. Buske, M.T. Vilhena, T. Tirabassi \& B. Bodmann. "Air pollution steady-state advection-diffusion equation: the general three-dimensional solution". Journal of Environmental Protection, 3(09) (2012), 1124.

[12] G.L. Diniz. "A mudança no habitat de populações de peixes: de rio a represa - o modelo matemático". Master's thesis, DMA, IMECC, UNICAMP, Campinas, SP, (1994).

[13] T.M.V.S. Lacaz. Análises de problemas populacionais intraespecíficos e interespecíficos com difusão densidade-dependente. PhD thesis, DMA, IMECC, UNICAMP, Campinas, SP, (1999).

[14] M.T. Koga. Dinâmica populacional da Mosca-dos-chifres (Haematobia Irritans) em um ambiente com competição: simulações computacionais. PhD thesis, FEEC, UNICAMP, Campinas, SP, (2015).

[15] J.R. Sibert, J. Hampton, D.A. Fournier \& P.J. Bills. "An advection-diffusion-reaction model for the estimation of fish movement parameters from tagging data, with application to skipjack tuna (katsuwonus pelamis)". Can. J. Fish. Aquat. Sci., 56(6) (1999), 925-938. 
[16] M.M. Salvatierra. "Modelagem matemática e simulação computacional da presença de materiais impactantes tóxicos em casos de dinâmica populacional com competição inter e intra-específica," Master's thesis, DMA, IMECC, UNICAMP, Campinas, SP, (2005).

[17] R.C. Sossae. A presença evolutiva de um material impactante e seu efeito no transiente populacional de espécies interativas. PhD thesis, DMA, IMECC, UNICAMP, Campinas, SP, (2003).

[18] D.C. Guaca. "Impacto ambiental em meios aquáticos: modelagem, aproximação e simulação de um estudo na baía de Buenaventura-Colômbia”. Master's thesis, DMA, IMECC, UNICAMP, Campinas, SP, (2015).

[19] L.C. Abreu. "Influência de poluentes sobre macroalgas na Baía de Sepetiba, RJ: modelagem matemática, análise numérica e simulações computacionais". Master's thesis, DMA, IMECC, UNICAMP, Campinas, SP, (2009).

[20] L. Torre, P.C.C. Tabares, F. Momo, J.F.C.A. Meyer \& R. Sahade. "Climate change effects on antarctic benthos: a spatially explicit model approach". Climatic Change, 141(4) (2017), 733-746.

[21] S. Pregnolatto. Mal-das-cadeiras em capivaras: estudo, modelagem e simulação de um caso. $\mathrm{PhD}$ thesis, DMA, IMECC, UNICAMP, Campinas, SP, (2002).

[22] M. Missio. Modelos de EDP integrados a lógica Fuzzy e métodos probabilísticos no tratamento de incertezas: uma aplicação a febre aftosa em bovinos. PhD thesis, DMA, IMECC, UNICAMP, Campinas, SP, (2008).

[23] J.M.R. Souza. "Estudo da dispersão de risco de epizootias em animais: o caso da influenza aviária". Dissertação de Mestrado, DMA, IMECC, UNICAMP, Campinas, SP, (2010).

[24] R.S. Cantrell \& C. Cosner. "On the effects of nonlinear boundary conditions in diffusive logistic equations on bounded domains". J. Differ. Equations, 231(2) (2006), 768-804.

[25] C. Cosner \& Y. Lou. "Does movement toward better environments always benefit a population?". J. Math. Anal. Appl., 277(2) (2003), 489-503.

[26] V. Méndez, D. Campos, I. Pagonabarraga, \& S. Fedotov. "Density-dependent dispersal and population aggregation patterns," J. Theor. Biol., 309 (2012), 113-120.

[27] N. Shigesada, K. Kawasaki \& H.F. Weinberger. "Spreading speeds of invasive species in a periodic patchy environment: effects of dispersal based on local information and gradient-based taxis". Jpn. J. Ind. Appl. Math., 32(3) (2015), 675-705.

[28] R.S. Cantrell \& C. Cosner. Spatial ecology via reaction-diffusion equations. Chichester: John Wiley \& Sons, (2004).

[29] B.P. Boudreau. Diagenetic Models and Their Implementation, vol. 505. Berlin: Springer Berlin, (1997).

[30] R.J. LeVeque. Finite difference methods for ordinary and partial differential equations: steady-state and time-dependent problems, vol. 98. Philadelphia: Siam, (2007).

\section{APPENDIX}

In this appendix we describe the calculation of numerical solutions for problem (2.1) with the general boundary condition (2.5), using second order Finite Difference formulas in spatial variables and Crank-Nicolson Method in the time variable [30]. Considering a square domain 
$\Omega=[0, L] \times[0, H]$, with partitions $\left\{x_{1}, \ldots, x_{n+1}\right\} \times\left\{y_{1}, \ldots, y_{n+1}\right\}$ for $\Omega$ and $\left\{t_{1}, \ldots, t_{m+1}\right\}$ for $I=\left[t_{0}, t_{f}\right]$, we use the notation $u\left(x_{i}, y_{j}, t_{k}\right)=u_{i, j}^{k}$ for $i, j=, 1 \ldots, n+1$, and $k=1, \ldots, m+1$. The difference formulas used were:

$$
\begin{aligned}
& \frac{\partial u_{i, j}^{k}}{\partial x} \approx \frac{u_{i+1, j}^{k}-u_{i-1, j}^{k}}{2 \Delta x}, \frac{\partial^{2} u_{i, j}^{k}}{\partial x^{2}} \approx \frac{u_{i+1, j}^{k}-2 u_{i, j}^{k}+u_{i-1, j}^{k}}{\Delta x^{2}}, \frac{\partial u_{i, j}^{n+\frac{1}{2}}}{\partial t} \approx \frac{u_{i, j}^{k+1}-u_{i, j}^{k}}{\Delta t} \\
& \frac{\partial u_{i, j}^{k}}{\partial y} \approx \frac{u_{i, j+1}^{k}-u_{i, j-1}^{k}}{2 \Delta y}, \frac{\partial^{2} u_{i, j}^{k}}{\partial y^{2}} \approx \frac{u_{i, j+1}^{k}-2 u_{i, j}^{k}+u_{i, j-1}^{k}}{\Delta y^{2}}, u_{i, j}^{n+\frac{1}{2}} \approx \frac{u_{i, j}^{k+1}+u_{i, j}^{k}}{2}
\end{aligned}
$$

Applying these formulas in (2.1) we obtain:

$$
\begin{aligned}
& \left(-\frac{\alpha \Delta t}{2 \Delta x^{2}}-\frac{v \Delta t}{4 \Delta x}\right) u_{i-1, j}^{k+1}+\left(-\frac{\alpha \Delta t}{2 \Delta y^{2}}+\frac{w \Delta t}{4 \Delta y}\right) u_{i, j-1}^{k+1} \\
+ & \left(1+\frac{\alpha \Delta t}{\Delta x^{2}}+\frac{\alpha \Delta t}{\Delta x^{2}}\right) u_{i, j}^{k+1}+\left(-\frac{\alpha \Delta t}{2 \Delta y^{2}}+\frac{w \Delta t}{4 \Delta y}\right) u_{i, j+1}^{k+1} \\
+ & \left(-\frac{\alpha \Delta t}{2 \Delta x^{2}}+\frac{v \Delta t}{4 \Delta x}\right) u_{i+1, j}^{k+1}=\left(\frac{\alpha \Delta t}{2 \Delta x^{2}}+\frac{v \Delta t}{4 \Delta x}\right) u_{i-1, j}^{k} \\
+ & \left(\frac{\alpha \Delta t}{2 \Delta y^{2}}+\frac{w \Delta t}{4 \Delta y}\right) u_{i, j-1}^{k}+\left(1-\frac{\alpha \Delta t}{\Delta x^{2}}-\frac{\alpha \Delta t}{\Delta x^{2}}\right) u_{i, j}^{k} \\
+ & \left(\frac{\alpha \Delta t}{2 \Delta y^{2}}-\frac{w \Delta t}{4 \Delta y}\right) u_{i, j+1}^{k}+\left(\frac{\alpha \Delta t}{2 \Delta x^{2}}-\frac{v \Delta t}{4 \Delta x}\right) u_{i+1, j}^{k}
\end{aligned}
$$

For points at the boundary, we need to apply the difference formulas on boundary condition (2.5). We then obtain, for any $k$, where $\vec{n}=\left(n_{1}, n_{2}\right)$ :

$$
\begin{aligned}
& -\frac{\alpha n_{1} \Delta t}{2 \Delta x} u_{i+1, j}^{k}+\frac{\alpha n_{1} \Delta t}{2 \Delta x} u_{i-1, j}^{k}-\frac{\alpha n_{2} \Delta t}{2 \Delta y} u_{i, j+1}^{k} \\
& \quad+\frac{\alpha n_{2} \Delta t}{2 \Delta y} u_{i, j-1}^{k}+\left(v n_{1}+w n_{2}-\beta\right) u_{i, j}^{k}=(\gamma-\beta) c .
\end{aligned}
$$

For each side of the rectangular domain, we have different normal vectors $\vec{n}$, which lead us to the following expressions, plugged in (4.1) for inexistent points of the mesh grid, for any $k$ :

$$
\begin{aligned}
& \vec{n}=(-1,0) \Rightarrow u_{i-1, j}^{k}=u_{i+1, j}^{k}-\frac{2 \Delta x}{\alpha}\left[(v+\beta) u_{i, j}^{k}+(\gamma-\beta) c\right] . \\
& \vec{n}=(0,-1) \Rightarrow u_{i, j-1}^{k}=u_{i, j+1}^{k}-\frac{2 \Delta y}{\alpha}\left[(w+\beta) u_{i, j}^{k}+(\gamma-\beta) c\right] . \\
& \vec{n}=(1,0) \Rightarrow u_{i+1, j}^{k}=u_{i-1, j}^{k}+\frac{2 \Delta x}{\alpha}\left[(v-\beta) u_{i, j}^{k}-(\gamma-\beta) c\right] . \\
& \vec{n}=(0,1) \Rightarrow u_{i, j+1}^{k}=u_{i, j-1}^{k}+\frac{2 \Delta y}{\alpha}\left[(w-\beta) u_{i, j}^{k}-(\gamma-\beta) c\right] .
\end{aligned}
$$


Equations (4.1) with corrections by (4.2) lead us to a linear system, with can be written as:

$$
\mathbf{A} \mathbf{u}^{k+1}=\mathbf{B} \mathbf{u}^{k}+\mathbf{f} .
$$

This system is then solved by the $L U$ factorization method, at time steps $k=1, \ldots, m+1$, with the initial condition defined at $k=1$. As each time step solution $\mathbf{u}^{k+1}$ is obtained, the system total mass $M\left(t_{k+1}\right)=\iint_{\Omega} u d A$ is calculated by Simpson's method. A summary of this procedure is given by the following algorithm.

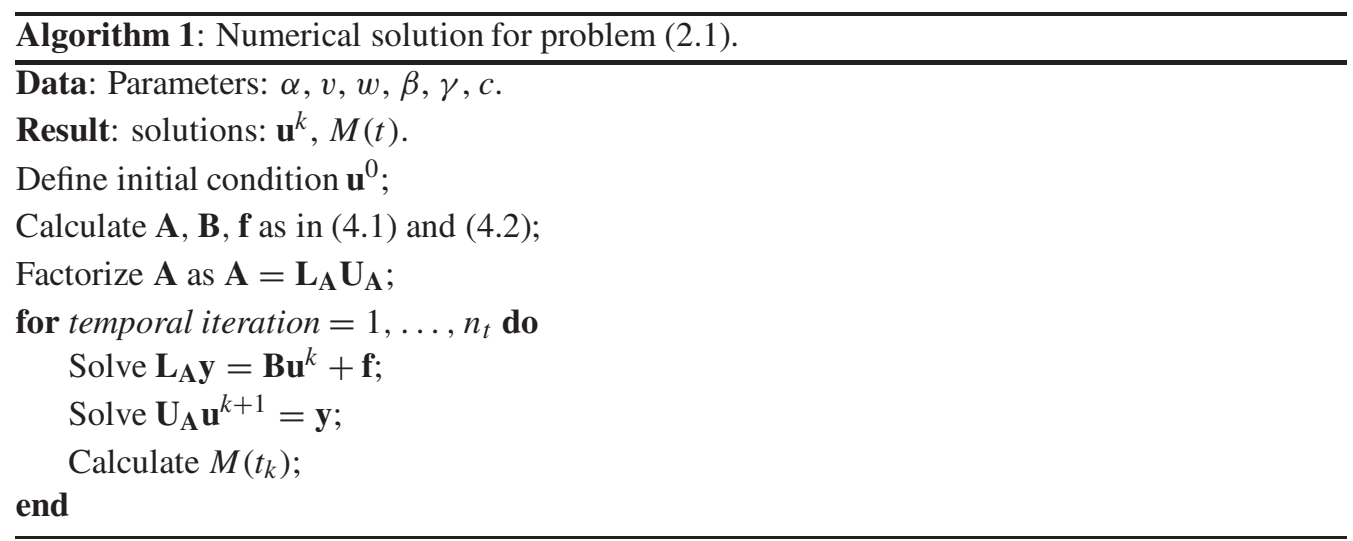

